\title{
BIBECHANA
}

ISSN 2091-0762 (Print), 2382-5340 (Online)

Journal homepage: http://nepjol.info/index.php/BIBECHANA

Publisher: Department of Physics, Mahendra Morang A.M. Campus, TU, Biratnagar, Nepal

\section{Visualization, formulation and intuitive explanation of iterative methods for transient analysis of series RLC circuit}

\author{
Jeevan Kafle ${ }^{1}$, Bhogendra Kumar Thakur ${ }^{1}$, Indra Bahadur Bhandari, ${ }^{2,3, *}$ \\ ${ }^{1}$ Central Department of Mathematics, Tribhuvan University, Kirtipur, Nepal \\ ${ }^{2}$ Central Department of Physics, Tribhuvan University, Kirtipur, Nepal \\ ${ }^{3}$ Department of Applied Sciences, Purwanchal Campus, Tribhuvan University, Dharan, Nepal \\ *E-mail: bhandari.indra@gmail.com
}

\section{Article Information:}

Received: September 14, 2020

\begin{abstract}
The time varying currents and voltages resulting from the sudden application of sources usually due to switching are transients. An RLC circuit is an electrical circuit consisting of a resistor, an inductor, and a capacitor, connected in series or in parallel. The transient response is dependent on the value of the different characteristics of damping factor (i.e., over damped, critically damped and under damped). We have computed the numerical solutions of second order differential equation with initial value problem (IVP) by using Explicit (Forward) Euler method, Third order Runge-Kutta (RK3) methods and Butchers fifth order RungeKutta (BRK5). The observation compares this numerical solution of ODEs obtained by above-mentioned methods among them with necessary visualization and analysis of the error. These iterative methods will be extended and implement to analyze the transient analysis of an RLC circuit. We have examined the superiority of those methods over one another. The Butchers fifth order RungeKutta (BRK5) method is found to be the best numerical technique to solve the transient analysis due to its high accuracy of approximations. Moreover, we consider the possibility of discussion and analyze above mentioned iterative methods in the cases of different characteristics of damping factor.
\end{abstract}

Accepted: November 08, 2020

Keywords:

Iterative methods

Transient analysis

Series RLC circuit

Damping Factor

Error analysis and DC source.

DOI: https://doi.org/10.3126/bibechana.v18i2.31208

This work is licensed under the Creative Commons CC BY-NC License. https://creativecommons.org/licenses/by-nc/4.0/

\section{Introduction}

While regarding the problem of mathematical modelling in the area of engineering, physics, aeronautics, medicine, environmental science, astronomy, chemistry, biology and many other applied fields, the role of differential equation is momentous [1, 2]. Meanwhile all of the differential model equation in real life appears to be nonlinear. On contrary, almost all differential equation 
encountered in course of modelling to be solved analytical, is out of the question [1]. Planetary motion under gravity, motion of simple pendulum, etc. are typical example of second order differential equation that won't solve analytically $[2,3]$. To wriggle out from such confounding situation one has to device a more rigorous alternative approach that would be simple and more pragmatic. One of such method is numerical method [3].

Transients are the momentary fluctuation of energy induced upon the electrical circuit due to the time varying currents and voltages resulting from the sudden application of sources. It usually occurs at the period of switching [4]. Sensitive component of the electrical circuitry such as millions of inductors, capacitors, resistors, transistor and diodes embedded inside integrated circuits are most vulnerable to this brief increase in current in voltage. An electrical system under the influence of transient condition rendered the system to an unsteady state. Thus, a transient analysis is to analyze the response of an electrical circuit under these unsteady state condition [5]. If the variables do not vary with respect to time, then the state of system is said to be in a steady state otherwise it is said to be in an unsteady state. Performance of any electrical circuit is measured by employing the methodology of transient analysis [16]. Although transients do occur in the most apparent electrical equipment such as charger of an android phone, electric mixture, CFL bulb, iron etc. but it is not at threatening level because components are adjusted enough to handle such ailments [7]. But transient occurring in the microscopic level inside a microprocessor, motherboard and memory card could be lethal to the existence of the system.

Suhag (2013) [4] conducted the transient analysis of second order RLC circuit and observed the response of the system by changing the conditions from one steady state value to another. He concluded RK method was very efficient in solving second order differential equations. Kee and Ranom (2018) [5] studied the transient analysis of series RLC circuit under underdamped, critically damped and overdamped conditions using RK-4 with different time step size h. The accuracy of RK-4 method is determined by gradually as the step size decimates. Thus, the improved RK-4 method by decreasing the step size $h$ or increasing the number of steps $n$ was a favorably suited method for solving transient analysis of electric circuit due to its high degree of accuracy and efficiency. Henry et al. (2018) [7] undertook the task of transient analysis of a secondorder RLC circuit by employing two numerical methods viz. Heun's method and Runge-Kutta method. They observed that the Heun's method reaches the stable limit first, thus, converges faster but the Runge-Kutta 4th order method proved to be more accurate. Thus Runge-Kutta method was recommended for transient analysis of complex electric circuits.

Ogbuka et al. (2008) [8] in their paper reiterated the need of a sound mathematical background and knowledge of basic laws of circuit analysis before handling the task of circuit analysis and they programmed the state equations of a sample complex circuit in two simulation software MATLAB and SIMPLORER to generate transient response. Deshpande (2014) [5] stated the source dc voltage applied in any circuit (RLC) constituting of energy storage device gives rise to transients and therefore estimation of the voltage was of significant importance for instrumentation and control purpose. Deshpande carried out the transient analysis to this circuit by employing Classical and Laplace Transformation method. Also, in cases of over damped, underdamped and critically damped conditions were evaluated significant for natural response of RLC series circuit. Laplace transform method had proved to be a better tool with respect to initialization condition in comparison to classical method. Ahamad and Charan (2019) [6] in order to solve initial value problem for fourth order ODEs presented fifth order Runge-Kutta method (RK5). Kamruzzaman and Nath (2018) [11] in their paper employed the Modified Euler and Runge-Kutta Methods to discuss the numerical solution of an ODE with IVP. They carried out the numerical 
simulation and compared the numerical solution with exact solution. Maffezzoni et al. (2007) [12] presented a novel approach to the accurate timedomain simulation of non-linear circuits that employs a class of high-order implicit Runge-Kutta (RK) method. The properties of stability and accuracy of these RK methods were briefly reviewed while the implementation in the flow of an analog simulator was described in detail.

In this work, we take a series RLC circuit with DC source which can be modelled with a second order ODE. For solving IVP of ODE and transient analysis, we first compared the three iterative method viz. Explicit Euler, Third order Runge-Kutta (RK3) and Butcher fifth order Runge-Kutta (BRK5). BRK5 method is observed to be the best method as it gives the least error compared to other two methods. BRK5 method is then chosen to analyze the three-damping condition of RLC circuit.

The approach of this paper is primarily analytical and comparative among the various numerical method for solving differential equation. Section-2 comprises of the theoretical approach and three iterative methods with the necessary numerical formulation. Section-3 depicts the simulation result and comparison of above-mentioned method. Section-4 culminate the conclusion and finding of this work.

\section{Theory}

A RLC circuit constitute of three electrical elements viz. a resistor, an inductor and a capacitor. It is a second order circuit. These circuits are the most popular as they are applied to construct oscillator and tuners of radio or audio receiver [4]. In the adjacent Fig. 1, the electrical elements resistor (R), inductor (L) and capacitor (C) are connected in series with the D.C. source $\left(V_{s}\right)$, which we call RLC series circuit. A circuit equation is drawn to undertake the analytical solution part of a transient analysis [5]. An ordinary electrical circuitry could contain thousands of components. So, any analytical solution in such RLC circuits is virtually impossible. In such scenario numerical methods could offer a great relief to the solution of system. Here, we used possible iterative methods to solve the circuit equation under the terms of transient analysis in an RLC circuit and compare different methods. The Kirchhoff's Voltage Law [5] is applied around the loop for $t>0$ in which the voltage source of the circuit in Fig. 1 is calculated by the following:

$$
V_{S}=V_{R}+V_{L}+V_{C}
$$

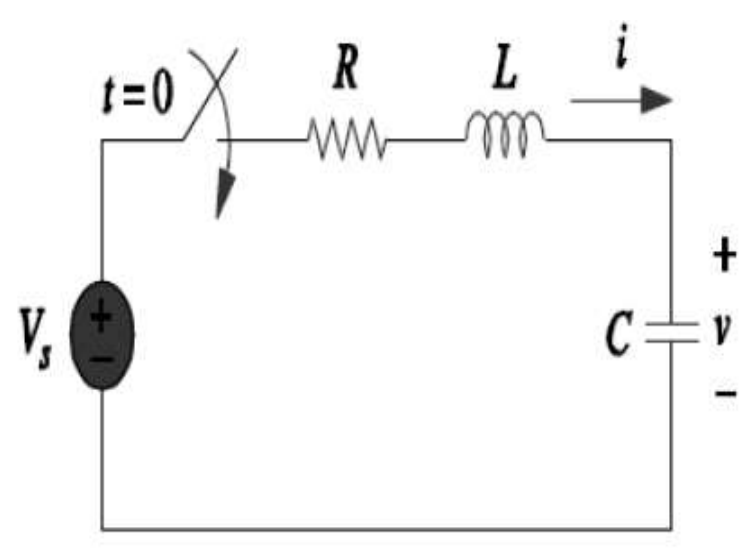

Fig. 1: RLC circuit [7].

The differential equations of the RLC in Fig. 1 is based on method of loop currents where the fundamental relationship between the current, and the individual circuit elements (Capacitance, Inductance, Resistance) are given by

$$
\begin{aligned}
& \text { Resistance }: v(t)=R i(t), \\
& \text { Capacitance }: i(t)=C \frac{d v}{d t}, \\
& \text { Inductance }: v(t)=L \frac{d i}{d t}
\end{aligned}
$$

The second-order differential equation of the RLC circuit with constant coefficients is written as [17]

$$
L \frac{d^{2} V}{d t^{2}}+R \frac{d V}{d t}+\frac{1}{C} V=\frac{V_{s}}{C} \quad \ldots \ldots \cdots
$$

The series RLC circuit is analyzed in order to determine its transient characteristics once the switch is closed. Equation (1) can be solved using different iterative methods [7].

The damping factor is responsible for amount by which the oscillation of a system gradually decreases with the time $(\boldsymbol{t})$. The transient response 
is dependent on the value of the damping factor $(\zeta)$ [7]. In RLC circuit, the damping factor is given by

$$
\zeta=\frac{\alpha}{\omega_{o}}
$$

where, $\boldsymbol{\alpha}=\frac{\boldsymbol{R}}{\mathbf{2} \boldsymbol{L}}$ (damping coefficient) and $\boldsymbol{\omega}_{\mathbf{0}}=$ $\frac{1}{\sqrt{L C}}$ (resonant frequency). Then

$$
\zeta=\frac{R}{2} \sqrt{\frac{C}{L}}
$$

The system is (i) over damped when $\zeta>1$, (ii) critically damped when $\zeta=1$, and (iii) under damped when $\zeta<1$ [4].

\section{Numerical methodologies}

In this section, we discuss different iterative method to solve initial value problems (IVP) for ordinary differential equations (ODE). First, we introduce Euler's method, the simplest method. We will provide details on algorithm development using the Euler method (explicit), Third order Ruge-Kutta Method (RK3) and Butcher's fifth order RungeKutta (BRK5) method [12, 13].

\section{Euler's method}

Consider one dimensional initial value problem (IVP)

$$
y^{\prime}(t)=f(t, y(t)), \quad y\left(t_{0}\right)=y_{0}
$$

The slope at the point $\left(\boldsymbol{t}_{\boldsymbol{n}}, \boldsymbol{y}_{\boldsymbol{n}}\right)$ is

$$
\begin{aligned}
\frac{y_{n+1}-y_{n}}{h} & \approx \frac{d y}{d t}=f\left(t_{n}, y_{n}\right) \\
\Rightarrow y_{n+1} & =y_{n} \\
& +h f\left(t_{n}, y_{n}\right) \ldots \ldots \ldots
\end{aligned}
$$

This formula (3) is Forward Euler's Method.

\section{Third order Runge-Kutta (RK3) method}

The third order Runge-Kutta method (RK3) is broadly used for solving initial value problems (IVP) for ordinary differential equation (ODE). The general formula for Runge-Kutta third order (RK3) method is shown below [15]

$$
y_{n+1}=y_{n}+h\left[\frac{k_{1}}{6}+\frac{2 k_{2}}{3}+\frac{k_{3}}{6}\right]
$$

where

$$
\begin{gathered}
k_{1}=f\left(t_{n}, y_{n}\right), \quad k_{2}=f\left(t_{n}+\frac{h}{2}, y_{n}+\frac{h}{2} k_{1}\right), \\
k_{3}=f\left(t_{n}+h, y_{n}-h k_{1}+2 h k_{2}\right)
\end{gathered}
$$

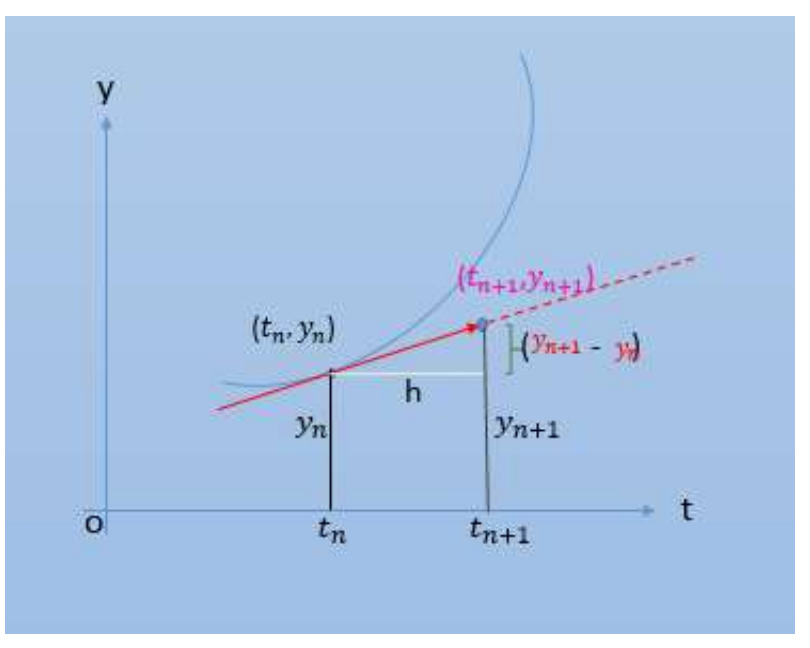

Fig. 2: Visualization of Euler method [14].

\section{Butchers fifth order Runge-Kutta (BRK5) method}

This method is distinguished by their order in the sense that agrees with Taylors series solution up to terms of $h^{r}$ where $r$ is the order of the method [7]. In this method, we consider the IVP (2). The method is shown below [13].

$$
\begin{gathered}
y_{n+1}=y_{n}+\frac{h}{90}\left(7 k_{1}+32 k_{3}+12 k_{4}+32 k_{5}\right. \\
\left.+7 k_{6}\right)
\end{gathered}
$$

where,

$$
\begin{gathered}
t_{n+1}=t_{n}+h, \quad k_{1}=f\left(t_{n}, y_{n}\right), k_{2}=f\left(t_{n}+\right. \\
\left.\frac{h}{4}, y_{n}+\frac{h}{4} k_{1}\right), \\
k_{3}=f\left(t_{n}+\frac{h}{4}, y_{n}+\frac{1}{8} h k_{1}+\frac{1}{8} h k_{2}\right), k_{4}= \\
f\left(t_{n}+\frac{h}{2}, y_{n}-\frac{1}{2} h k_{2}+h k_{3}\right), \\
k_{5}=f\left(t_{n}+\frac{3 h}{4}, y_{n}+\frac{3}{16} h k_{1}+\frac{9}{16} h k_{4}\right), k_{6}= \\
f\left(t_{n}+h, y_{n}-\frac{3}{7} h k_{1}+\frac{2}{7} h k_{2}+\frac{12}{7} h k_{3}-\frac{12}{7} h k_{4}+\right. \\
\left.\frac{8}{7} h k_{5}\right)
\end{gathered}
$$

We can extend above discussed iterative methods for the solution of higher order initial value 
problems (IVP) for ordinary differential equations (ODE) and system of initial value problems (IVP) for ordinary differential equations (ODE) $[3,16]$.

\section{Numerical formulation of RLC circuit}

Let,

$$
v=x \text { and } \frac{d v}{d t}=\frac{y}{C} \quad \ldots \ldots \ldots
$$

then equation (1) becomes

$$
\frac{d y}{d t}=\frac{V_{s}-x-R \times y}{L} \quad \ldots \ldots \ldots
$$

Hence equation (6) and (7) form a system of first order differential equation [7].

Let,

$$
f=\frac{d v}{d t}=\frac{y}{C} \text { and } g=\frac{d y}{d t}=\frac{V_{s}-x-R \times y}{L}
$$

\section{Euler's method for RLC circuit}

$$
\begin{aligned}
& x_{i+1}=x_{i}+h f\left(t_{i}, x_{i}, y_{i}\right) \\
& y_{i+1}=y_{i}+h g\left(t_{i}, x_{i}, y_{i}\right)
\end{aligned}
$$

where $h$ is the time step size.

\section{RK3 method for RLC circuit}

where

$$
\begin{gathered}
x_{i+1}=x_{i}+\left(f_{1}+4 f_{2}+f_{3}\right) / 6 \\
y_{i+1}=y_{i}+\left(g_{1}+4 g_{2}+g_{3}\right) / 6
\end{gathered}
$$

$$
\begin{gathered}
f_{1}=h f\left(t_{i}, x_{i}, y_{i}\right), g_{1}=h g\left(t_{i}, x_{i}, y_{i}\right), \\
f_{2}=h f\left(\left(t_{i}+\frac{h}{2}\right),\left(x_{i}+\frac{f_{1}}{2}\right),\left(y_{i}+\frac{g_{1}}{2}\right)\right), \\
g_{2}=h g\left(\left(t_{i}+\frac{h}{2}\right),\left(x_{i}+\frac{f_{1}}{2}\right),\left(y_{i}+\frac{g_{1}}{2}\right)\right), \\
f_{3}=h f\left(\left(t_{i}+h\right),\left(x_{i}-f_{1}+2 f_{2}\right),\left(y_{i}-g_{1}+\right.\right. \\
\left.\left.2 g_{2}\right)\right) \text { and } \\
g_{3}=h g\left(\left(t_{i}+h\right),\left(x_{i}-f_{1}+2 f_{2}\right),\left(y_{i}-g_{1}\right.\right. \\
\left.\left.+2 g_{2}\right)\right)
\end{gathered}
$$

\section{Formulating BRK5 method for RLC circuit}

$$
\begin{aligned}
x_{i+1}=x_{i} & +\left(7 f_{1}+32 f_{3}+12 f_{4}+32 f_{5}\right. \\
& \left.+7 f_{6}\right) / 90 \\
y_{i+1}=y_{i} & +\left(7 g_{1}+32 g_{3}+12 g_{4}+32 g_{5}\right. \\
& \left.+7 g_{6}\right) / 90
\end{aligned}
$$

where

$f_{1}=h f\left(t_{i}, x_{i}, y_{i}\right), \quad g_{1}=h g\left(t_{i}, x_{i}, y_{i}\right), \quad f_{2}=$ $h f\left(\left(t_{i}+\frac{h}{4}\right),\left(x_{i}+\frac{f_{1}}{4}\right),\left(y_{i}+\frac{g_{1}}{4}\right)\right)$,

$$
\begin{aligned}
& g_{2}=h g\left(\left(t_{i}+\frac{h}{4}\right),\left(x_{i}+\frac{f_{1}}{4}\right),\left(y_{i}+\frac{g_{1}}{4}\right)\right), f_{3} \\
& =h f\left(\left(t_{i}+\frac{h}{4}\right),\left(x_{i}+\frac{f_{1}+f_{2}}{8}\right),\left(y_{i}\right.\right. \\
& \left.\left.+\frac{g_{1}+g_{2}}{8}\right)\right) \\
& g_{3}=h g\left(\left(t_{i}+\frac{h}{4}\right),\left(x_{i}+\frac{f_{1}+f_{2}}{8}\right),\left(y_{i}+\frac{g_{1}+g_{2}}{8}\right)\right) \text {, } \\
& f_{4}=h f\left(\left(t_{i}+\frac{h}{2}\right),\left(x_{i}-\frac{f_{2}}{2}+f_{3}\right),\left(y_{i}-\frac{g_{2}}{2}+g_{3}\right)\right), \\
& g_{4}=h g\left(\left(t_{i}+\frac{h}{2}\right),\left(x_{i}-\frac{f_{2}}{2}+f_{3}\right),\left(y_{i}-\frac{g_{2}}{2}+g_{3}\right)\right) \text {, } \\
& f_{5}=h f\left(\left(t_{i}+\frac{3 h}{4}\right),\left(x_{i}+\frac{3 f_{1}}{16}+\frac{9 f_{4}}{16}\right),\left(y_{i}+\frac{3 g_{1}}{16}+\frac{9 g_{4}}{16}\right)\right) \text {, } \\
& g_{5}=h g\left(\left(t_{i}+\frac{3 h}{4}\right),\left(x_{i}+\frac{3 f_{1}}{16}+\frac{9 f_{4}}{16}\right),\left(y_{i}+\frac{3 g_{1}}{16}+\frac{9 g_{4}}{16}\right)\right) \text {, } \\
& f_{6}=h f\left(\left(t_{i}+h\right),\left(x_{i}-\frac{3 f_{1}-2 f_{2}-12 f_{3}+12 f_{4}-8 f_{5}}{7}\right), \quad\left(y_{i}-\right.\right. \\
& \left.\left.\frac{3 g_{1}-2 g_{2}-12 g_{3}+12 g_{4}-8 g_{5}}{7}\right)\right), g_{6}=h g\left(\left(t_{i}+h\right),\left(x_{i}\right.\right. \\
& \left.\left.\frac{3 f_{1}-2 f_{2}-12 f_{3}+12 f_{4}-8 f_{5}}{7}\right),\left(y_{i}-\frac{3 g_{1}-2 g_{2}-12 g_{3}+12 g_{4}-8 g_{5}}{7}\right)\right)
\end{aligned}
$$

\section{Results and Discussion}

In the following sub section, we compare numerical solution of RLC circuits by above discussed numerical methods with exact solution and analyze the different characteristics of damping factor by using best superior iterative method.

\subsection{Comparison between analytical solution and numerical methods}

In this experiment, the simulation time for all the conditions is from $0 \mathrm{~s}$ to $20 \mathrm{~s}$ with the step size of $h=0.1 \mathrm{~s}$. Moreover, we consider source voltage $\left(V_{S}\right)=6 \mathrm{~V}$, resistance $(R)=1 \Omega$, inductance $(L)=1$ $H$ and capacitance $(C)=0.25 F$ [7] for the simulation result of RLC circuit. Fig. 3 describe simulation results of numerical solution of RLC circuit and exact solution under above conditions. In order to compare the accuracy of the numerical methods, the computed points of the three numerical methods are taken at the specific time. The computed point of each method is compared with the analytic solution. From the above Fig. 3, we observed that the approximate solution curve obtained from the Butcher fifth order Runge-Kutta (BRK5) method is converging faster towards the analytical solution in 
comparison with the other two methods. This shows that there is less error between BRK5 and analytical solution in comparison with other two methods (Table 1). Table 1 contain details of simulated results obtained for Iterative methods and exact solution with Error in different time slices. Hence, we concluded that the BRK5 method is more efficient method to approximate the solution of RLC circuit.

So, in the following section, we consider three damping cases (i.e., underdamped, critically damped and over damped) for Butcher fifth order RungeKutta (BRK5) method. In Fig. 4, we simulate the results under discussed above condition with different time slices.

\subsection{Characteristics of Damping Factor by using BRK5 method}

The different damping condition of RLC circuit and their characteristics are as discussed below:

Condition 1: Underdamped (i. e., $\zeta<1$ )

In this condition, the resistance, $\mathrm{R}$ must be less than $4 \Omega$ in order to achieve underdamped response. In order to obtain the best numerical method for transient analysis of RLC circuit, three numerical methods are compared with the analytical solution. The three numerical methods mentioned are Euler method, RK3 method and BRK5 method. The experiment is conducted by taking $1 \Omega$ as the value of resistor, $\mathrm{R}$ for the circuit $[4,5,7]$.

\section{Condition 2: Critically damped (i. e. , $\zeta=1$ )}

In critically damped response, the resistance, $\mathrm{R}$ must be equal to $4 \Omega$ in order to achieve the critically damped condition.

\section{Condition 3: Overdamped (i. e. , $\zeta>1$ )}

In condition 3 , the resistance, $\mathrm{R}$ must be more than $4 \Omega$ in order to achieve overdamped response. The experiment is conducted by taking $6 \Omega$ as the value of resistor, $\mathrm{R}$ for the circuit. Table 2 contain all the condition for different damping factor. By the variation of the resistance $(R)$, we can get three different damping conditions.

In Fig. 4, we observed that the under damped responses are a decaying oscillation decays at a rate determined by the attenuation ( $\alpha$ ) by using BRK5

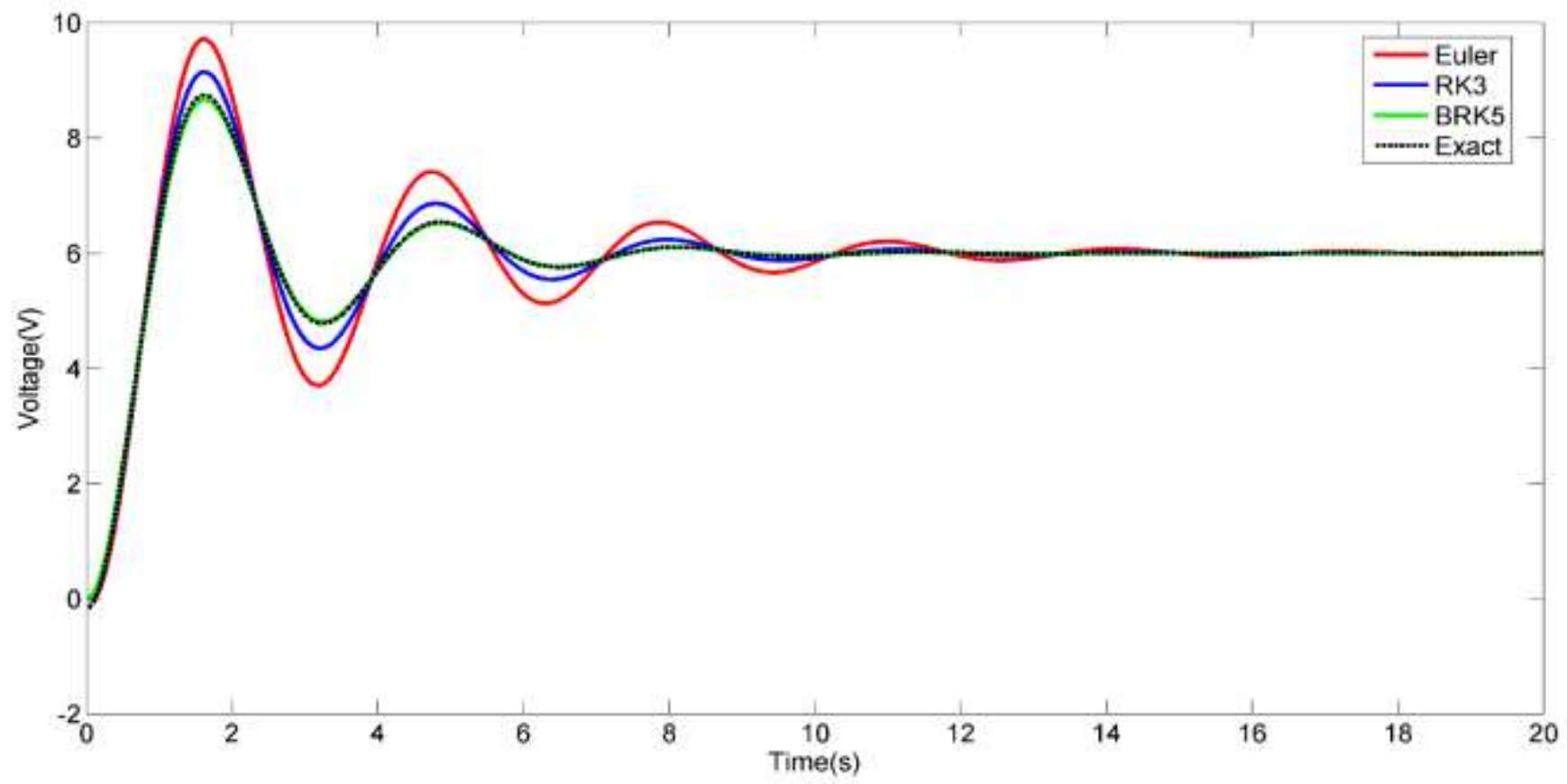

Fig. 3: Comparison of Euler, RK3, BRK5 and Analytical Solution. 
method. The exponential decay describes the envelope of the oscillation. Here, the oscillation is sinusoidal with exponentially decaying amplitude. The critically damped response represents the circuit response that decays in the fastest possible time without going into oscillation. This consideration is important in control systems where it is required to reach the desired state as quickly as possible without overshooting. The overdamped response is a decay of the transient current without oscillation, which reach the stable state slower than the critically damped case. The quantitative description of numerical of solution under three damping condition by using BRK5 method is detailed in Table 3.

Table 1: Simulated results obtained for Iterative methods and exact solution with error.

\begin{tabular}{|c|c|c|c|c|c|c|c|}
\hline \multirow{2}{*}{$\begin{array}{l}\text { Time }(\mathrm{t}) \\
\text { (seconds) }\end{array}$} & \multicolumn{2}{|c|}{ Euler } & \multicolumn{2}{|c|}{$R K_{3}$} & \multicolumn{2}{|c|}{$B R K_{5}$} & \multirow{2}{*}{$\begin{array}{c}\text { Exact } \\
\text { Voltage }(\mathrm{V})\end{array}$} \\
\hline & Voltage(V) & $E_{R}\left(10^{-3}\right)$ & Voltage(V) & $E_{R}\left(10^{-3}\right)$ & Voltage(V) & $E_{R}\left(10^{-3}\right)$ & \\
\hline 0.0 & 0.000000 & 0.000000 & 0.000000 & 0.000000 & 0.000000 & 0.000000 & 0.000000 \\
\hline 0.1 & 0.000000 & 4.378000 & 0.116000 & 1.622000 & 0.115714 & 1.336000 & 0.114378 \\
\hline 0.2 & 0.240000 & 3.654000 & 0.444083 & 0.429000 & 0.443657 & 3.000000 & 0.443654 \\
\hline 0.3 & 0.696000 & 6.189000 & 0.951609 & 0.580000 & 0.951190 & \begin{tabular}{|c|}
9.990000 \\
\end{tabular} & 0.952189 \\
\hline 0.4 & 1.336800 & 5.764000 & 1.602239 & 0.325000 & 1.601968 & 5.960000 & 1.612564 \\
\hline 0.5 & 2.125680 & 1.791000 & 2.357671 & 8.800000 & 2.357671 & 8.800000 & 2.366471 \\
\hline 0.6 & 3.022200 & 7.630000 & 3.179275 & 0.555000 & 3.179643 & 1.870000 & 3.189830 \\
\hline 0.7 & 3.984041 & 1.851000 & 4.029593 & 4.701000 & 4.030393 & \begin{tabular}{|l|}
501000 \\
\end{tabular} & 4.004892 \\
\hline 0.8 & 4.968810 & 4.868000 & 4.873643 & 0.299000 & 4.874908 & 9.034000 & 4.883942 \\
\hline 0.9 & 5.935740 & 3.419000 & 5.680016 & 2.305000 & 5.681740 & 5.810000 & 5.692321 \\
\hline 1.0 & 6.847225 & 2.573000 & 6.421720 & 2.932000 & 6.423867 & 7.850000 & 6.424652 \\
\hline
\end{tabular}

Table 2: The values of electrical elements of series RLC circuit for three conditions [5].

\begin{tabular}{|l|c|l|c|}
\hline \multirow{2}{*}{ Element } & \multicolumn{2}{|c|}{ Value } \\
\cline { 2 - 4 } & Condition 1 & Condition 2 & Condition 3 \\
\hline Resistor & Underdamped & Critically damped & Overdamped \\
\hline DC (Vs) & $6 \mathrm{~V}$ & $6 \mathrm{~V}$ & $6 \mathrm{~V}$ \\
\hline Resistor R & $<4 \Omega$ & $4 \Omega$ & $>4 \Omega$ \\
\hline Inductor L & $1 \mathrm{H}$ & $1 \mathrm{H}$ & $1 \mathrm{H}$ \\
\hline Capacitor C & $0.25 \mathrm{~F}$ & $0.25 \mathrm{~F}$ & $0.25 \mathrm{~F}$ \\
\hline
\end{tabular}




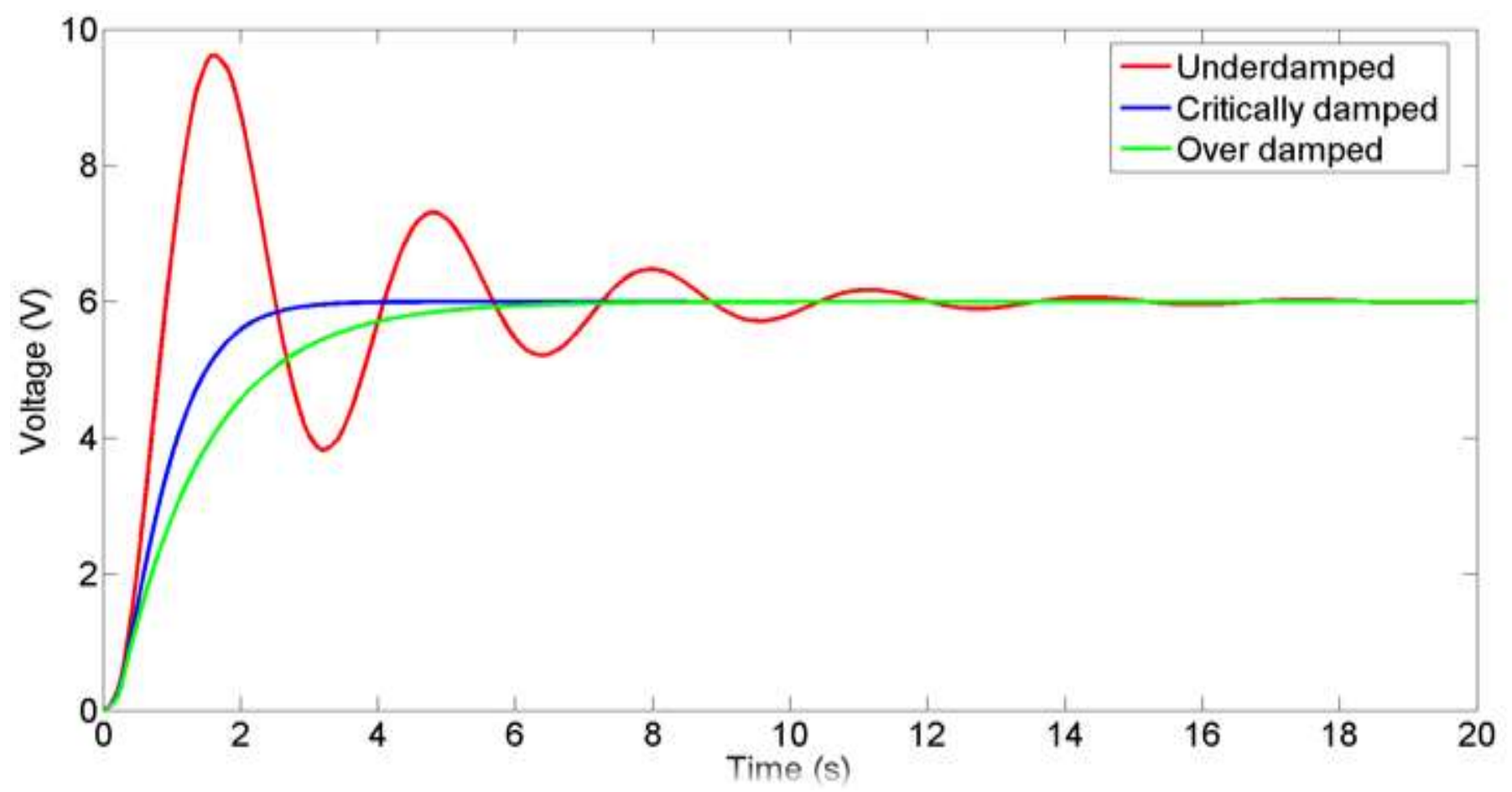

Fig. 4 : Comparison of three different damping condition by using BRK5.

Table 3: Values of three conditions of BRK5 method.

\begin{tabular}{|c|c|c|c|}
\hline \multirow{2}{*}{ Time (s) } & \multicolumn{3}{|c|}{ Values of BRK5 Method } \\
\cline { 2 - 4 } & Underdamped & Critically damped & Overdamped \\
\hline 0.0 & 0.000000 & 0.000000 & 0.000000 \\
\hline 0.1 & 0.115714 & 0.105139 & 0.098897 \\
\hline 0.2 & 0.443657 & 0.369312 & 0.330084 \\
\hline 0.3 & 0.951190 & 0.731409 & 0.626944 \\
\hline 0.4 & 1.601968 & 1.147248 & 0.950945 \\
\hline 0.5 & 2.357643 & 1.585448 & 1.280130 \\
\hline 0.6 & 3.179643 & 2.024237 & 1.602290 \\
\hline 0.7 & 4.030393 & 2.449004 & 1.910937 \\
\hline 0.8 & 4.874908 & 2.850415 & 2.202915 \\
\hline 0.9 & 5.681740 & 3.222979 & 2.476992 \\
\hline 1.0 & 6.423867 & 3.563965 & 2.733027 \\
\hline
\end{tabular}

\section{Conclusion}

The transient analysis of electrical circuit is analyzed using analytical method, Euler (Explicit) Method, RK3 Methods and BRK5 Method. With the usage of computational software, the process of obtaining results of transient analysis is done systematically and conveniently. It has been observed that the BRK5 method is very efficient in solving second order differential equations. Thus, we can conclude that by carrying out the transient 
analysis of a system, we can find out the response of the system by changing the conditions from one steady state value to another. Our observation clearly shows that the under damped decay is oscillatory and exponential. However, the other two are non-oscillatory exponential decay. The decay in critically damped case is observed faster than in over damped case. This response helps in designing a system, which meets our requirements, and we can further optimize the time domain parameters of the system.

\section{References}

[1] N. Ahamad and S. Charan, Study of numerical solution of fourth order ordinary differential equations (ODEs) by fifth order Runge-Kutta method, International Journal of Science, Engineering and Technology 6 (2019) 230-237. https://doi.org/10.32628/IJSRSET196142

[2] A. Kendall, H. Weimin and S. David, Numerical Solution of Ordinary Differential Equations, University of Iowa, Iowa City, A John Wiley \& Sons, Inc., Publication (2009).

[3] Md. J. Hossain, Md. S. Alam and Md. B. Hossain, A study on numerical solutions of second order initial value problems (IVP) for ordinary differential equations with fourth order and butchers fifth order Runge-Kutta methods, American Journal of Computational and Applied Mathematics 7(5) (2017) 129-137. https://doi.org/10.5923/j.ajcam.20170705.02

[4] A. Suhag, Transient analysis of electrical circuits using Runge-Kutta method and its application, International Journal of Scientific and Research Publications 3 (2013) 486-490.

[5] T. A. Kee, R. Ranom, Comparison of numerical technique in solving transient analysis of electrical circuits, ARPN Journal of Engineering and Applied Sciences 13(1) (2018) 314-320.

[6] A. Balota, S. Grebovic, Importance of lightning activity monitoring systems in transient analysis on wind turbine blade, Fourth International Symposium on Environmental Friendly Energies and Applications, 1(2016) 1-6. https://doi.org/10.1109/EFEA.2016.7748804
[7] O. Henry, B. Albert, I. Justice, Application of numerical methods in transient analysis, IJSR 8(5) (2019) 2108-2112.

[8] C.U. Ogbuka, O.I. Okoro, M.U. Agu, Solving electrical circuits transient problems with MATLAB® and SIMPLORER®. Pacific Journal of Science and Technology 9 (2008) 149-154.

[9] A.S. Deshpande, Transient analysis of RLC series circuit to step voltage by engineering method, International Journal of Computational and Applied Mathematics 9 (2014) 63-70.

[10] Md. Kamruzzama, M.C. Nath, A comparative study on numerical solution of initial value problem by using Euler's method, modified Euler's method and Runge-Kutta method, Journal of Computer and Mathematical Sciences 9(5) (2018)493-500. https://doi.org/10.29055/jcms/784

[11] P. Maffezzoni, L. Codecasa, D. D'Amore, Timedomain simulation of nonlinear circuits through implicit Runge-Kutta methods, Institute of Electrical and Electronics Engineers Transactions on Circuits and Systems 54(2) (2007) 391-400. https://doi.org/10.1109/TCSI.2006.887476

[12] W. E. Boyce and R. C. Di Prima, Elementary Differential Equations and Boundary Value Problems 10 (2012).

[13] J.C. Butcher, Numerical methods for ordinary differential equations, second edition, John Wiley and Sons, Ltd. (1933).

[14] Holistic numerical methods committed to Bringing Numerical Methods to the STEM Undergraduate.

https://nm.mathforcollege.com/

[15] C. Senthilnatha, A Numerical solutions of initial value problems for ordinary differential equations with Euler and higher order of Runge-Kutta methods using Matlab. International Journal of Engineering Science Invention 7(4) (2018) 25-31.

[16] Md. B. Hossain, Md. J. Hossain, Md. M. Miah and Md. S. Alam, A comparative study on fourth order and Butchers fifth order Runge-Kutta methods with third order initial value problem, Applied and Computational Mathematics 6(6) (2017) 243-253.

[17] J. Santiago, Circuit Analysis For Dummies, John Wiley \& Sons (2013). 